\title{
HTLV-1-Associated Myelopathy/Tropical Spastic Paraparesis Overlapping Ossification of the Ligamentum Flavum
}

\author{
Guilherme Gago ${ }^{1}$ Luidia Varrone Giacomini ${ }^{1}$ Frederico de Lima Gibbon ${ }^{2}$ \\ Euripedes Gomes de Carvalho Neto ${ }^{1}$ Fernando Kowacs ${ }^{1}$ Nelson Pires Ferreira ${ }^{1}$
}

\author{
${ }^{1}$ Hospital São José, Complexo Hospitalar Santa Casa de Misericórdia \\ de Porto Alegre, Porto Alegre, Rio Grande do Sul, Brazil \\ ${ }^{2}$ School of Medicine, Universidade Católica de Pelotas, Pelotas, \\ Rio Grande do Sul, Brazil
}

\author{
Address for correspondence Frederico de Lima Gibbon, MS, \\ Universidade Católica de Pelotas, Street Av. Major Carlos Pinto, 123, \\ Casa 14, Pelotas, Rio Grande, Brazil \\ (e-mail: fredericogibbon@gmail.com).
}
Abstract
Keywords
- chronic myelopathy
- tropical spastic paraparesis
- ossification of the ligamentum flavum

HTLV-1-associated myelopathy/tropical spastic paraparesis (HAM/TSP) is an important cause of nontraumatic and noncompressive chronic myelopathy which generally has no improvement regardless of treatment. On the other hand, ossification of the ligamentum flavum (OLF) is also a cause of myelopathy; however, it can improve in some cases when surgical treatment is well applied. In this case report, we presented a case of a patient with HAM/TSP overlapping OLF which showed some improvement with surgical treatment.

\section{Introduction}

Human T-lymphotropic virus 1 (HTLV-1) is a retrovirus that infects $\mathrm{T}$ lymphocytes. The two main and best described repercussions of HTLV-1 are adult T cell leukemia/lymphoma (ATL) and HTLV-1-associated myelopathy/tropical spastic paraparesis (HAM/TSP). Approximately $1 \%$ of HTLV-1-infected people develop an HAM/TSP which is a progressive disease of the central nervous system (CNS) and the leading cause of nontraumatic and noncompressive chronic myelopathy in many endemic countries. ${ }^{1}$

Ossification of the ligamentum flavum (OLF) usually occurs at the lower thoracic level and is a well-established cause of myelopathy, radiculopathy, and cauda equina syndrome which may require surgical intervention. ${ }^{2}$

We presented the case of a patient with overlapping HAM/ TSP and OLF who benefited from surgical treatment.

\section{Case Report}

Our patient was a 25-year-old female with slow-progressing paraparesis beginning at 13 years of age, leading to inability

DOI https://doi.org/ $10.1055 / \mathrm{s}-0040-1710106$ ISSN 2277-954X. to walk at 19 years of age. Her previous pathological history was without particularities. The patient had a family history of HTLV (father) myelopathy, which was diagnosed around her father's 30 years of age. In view of the above findings, the HTLV serology collection (enzyme-linked immunosorbent assay [ELISA]) was requested from the basic health system, which was reagent HTVL-1.

On admission, the patient had symmetrical pyramidal release syndrome affecting the lower limbs symmetrically, with spastic paraparesis (MMRC 2) and hyperreflexia; also, the latter occurred in the upper limbs. In addition, she also reported lower limb hypoesthesia without sensory level and urinary incontinence. Other aspects of the physical examination did not show any particularity.

Confirmation of HTLV-1 positivity was performed by Western Blot. The patient underwent imaging and laboratory tests for myelopathy. Laboratory screening for anti-HIV, VDRL, vitamin B12, and serum copper was negative. Magnetic resonance imaging (MRI) of the head did not show any changes, but the MRI of the spine showed ossification of the ligamentum flavum at the T5-T6 level, measuring $1.6 \mathrm{~cm}$ in longitudinal length ( $\boldsymbol{- \text { Fig. }} \mathbf{1}$ ). At this level, the spinal cord 


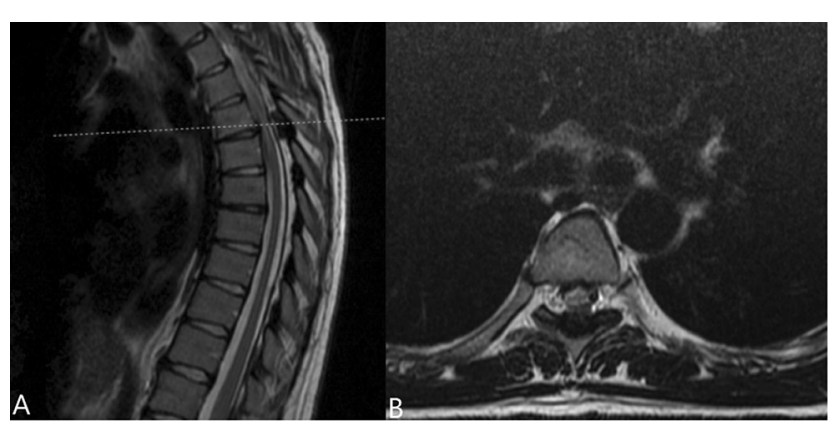

Fig. $1 \mathrm{MRI}$ of the thoracic spine, T2-weighted, showing ossification of the ligamentum flavum at the T5-T6 level. (A) showing the sagittal view and (B) showing the axial view.

presented signal thinning and hyperintensity at $\mathrm{T} 2$, probably representing myelomalacia.

After discussing the case with the neurology team, we decided to perform a decompressive laminectomy. The patient underwent laminectomy to treat spinal cord compression. At the 3-month follow-up, the paraparesis remained the same compared with the preoperative period, but showed improvement in lower limb sensitivity and urinary incontinence.

\section{Discussion}

The neurological manifestation of HTLV-1 is myelopathy, although it is rare. Symptoms usually begin approximately in the fifth decade of life. The presentation usually is subacute or chronic with paresis of the lower limbs, initially unilateral and later progressing to both lower limbs. In addition, there may be hyperreflexia, extensor plantar reflex, low-back pain, and sensory and urinary disorders. Olindo et $\mathrm{al}^{3}$ found that the mean time to progression from pararesis to wheelchair use was 21 years. However, our patient had a much shorter progression time than this, approximately 6 years. In the literature, there are some factors associated with higher risk of developing lifelong HAM/TSP, such as proviral load, genetic of the virus and of the host, and age. In addition, the main factors related to rapid progression disease are advanced age at disease onset, age above 50 years, and proviral load. ${ }^{1}$ Unfortunately, we had no access to the viral load of our patient which could explain why our patient progressed so quickly. However, we found another element which that could have contributed to the rapid progression disease, the OLF.
The OLF is also not a common disease and is closely related to the Japanese population. Its cause is not well-defined, but both systemic and local factors seem to be involved, such as heredity, abnormal metabolism of carbohydrate, abnormal metabolism of calcium, abnormal secretion of gender hormone, and degeneration of the ligament. ${ }^{2}$ Treatment of OLF may be expectant or surgical, depending on the clinical presentation of the patient. Surgical intervention is usually necessary and shows good results when the patient presents symptoms of spinal cord or root compression.

In our case, when the patient came to our service, she already showed signs of advanced chronic myelopathy. We identified that there was an overlap of HAM/TSP and OLF. Although HAM/TSP is a chronic and progressive disease, it is known that patients with OLF can considerably regress their symptoms with decompression surgery. Due to this and also the history of rapidly progressing paraparesis, we concluded that OLF could have been an important aggravating factor of the clinical condition of the patient. Thus, decompression surgery could result in some improvement in quality of life. Although the motricity of the patient showed no short-term improvement, improved of the lower limbs sensitivity and bladder dysfunction were sufficient to result in improved quality of life for the patient.

\section{Conclusion}

This case highlighted the importance of adequate screening for cases of chronic myelopathy (irrespective of its relation with HTLV). Although HAM/TSP is a chronic and progressive disease, most of the symptoms of the patient were probably not related to HAM/TSP, but to OLF.

\section{Conflict of Interest}

None declared.

\section{References}

1 Bangham CRM, Araujo A, Yamano Y, Taylor GP. HTLV-1associated myelopathy/tropical spastic paraparesis. Nat Rev Dis Primers 2015;1(1):15012

2 Hirabayashi S. Ossification of the ligamentum flavum. Spine Surg Relat Res 2017;1(4):158-163

3 Olindo S, Cabre P, Lézin A, et al. Natural history of human T-lymphotropic virus 1-associated myelopathy: a 14-year follow-up study. Arch Neurol 2006;63(11):1560-1566 